\title{
Bacteriology and antibiogram of pathogens from wound infections at Dessie Laboratory, North East Ethiopia
}

\author{
MULUGETA K. AZENE1* ${ }^{*}$ and BAYEH A. BEYENE² \\ ${ }^{1}$ Department of Biology, Science College, Bahir Dar University, P.O. Box 79, Bahir Dar, Ethiopia \\ ${ }^{2}$ Department of Microbiology, Parasitology and Immunology, College of Medicine and Health Sciences, \\ Bahir Dar University, P.O. Box 79, Bahir Dar, Ethiopia
}

\begin{abstract}
Wound infections result in sepsis, limb loss, long hospital stays, higher costs, and are responsible for significant human mortality and morbidity worldwide. The aim of this study was to investigate the profile of pathogens cultured from wound infection and determine the antimicrobial susceptibility patterns. A retrospective analysis of bacterial pathogens and their antimicrobial susceptibility was done on wound swab samples that have been cultured at Dessie Regional Laboratory from 2003 to 2010. Antimicrobial susceptibility tests were done using disc diffusion technique as per the standard of Kirby-Bauer method. The mean age of male and female patients was 31.2 and 29.8 years, respectively with male to female ratio of 1:1.6. Out of 599 wound swab samples analyzed, 422 (70.5\%) were culture positive. A total of 500 bacteria from 422 positive cases were identified. Seventy eight $(18.5 \%)$ of the culture had double infections. Staphylococcus aureus was the most frequently isolated pathogen which accounted for 208 (41.6\%) of isolates followed by Pseudomonas spp. 92 (18.4\%), E. coli 82 (16.4\%), Proteus spp. 55 (11.0\%), Enterobacter spp. 21 (4.2\%), and Citrobacter spp. 21 (4.2\%), Klebsiella spp. 12 (2.4\%) and Coagulate negative staphylococcus 9 (1.8\%). Amoxicillin had the highest resistance rate $78.9 \%$, followed by tetracycline $76.1 \%$ and erythromycin $(63.9 \%)$. The sensitivity rates of norfloxacin, ciprofloxacin and gentamicin were $95.1 \%, 91.8 \%$ and $85 \%$, respectively. The overall multiple antimicrobial resistances rate was $65.2 \%$ and only $13 \%$ of the isolates were sensitive to all antimicrobial agents tested. The most frequently isolated bacteria were sensitive to ciprofloxacin, gentamicin, cloxacillin and norfloxacin. These antimicrobials are considered as appropriate antimicrobials for empirical treatment of wound infections. Periodic surveillance of aetiology and drug susceptibility both in the community and hospital settings is recommended.
\end{abstract}

Key words: wound infection, antimicrobial resistance, Ethiopia

\section{Introduction}

Wound infections are responsible for significant human mortality and morbidity worldwide (Green $\mathcal{E}$ Wenzel, 1977). Wound infection results in sepsis, limb loss, long hospital stays and higher costs (Bowler et al., 2001; Branom, 2002; Cutting \& White, 2004). Wound infections are caused by both gram-positive and gram-negative bacteria, especially by Staphylococcus aureus,

\footnotetext{
* Correspondence: Mulugeta Kibret; E-mail: mulugetanig@gmail.com
} 
Escherichia coli, and Pseudomonas aeruginosa (Bowler \& Davies, 1999; Larson, 2002). Studies conducted in Ethiopia have shown that wound infections are caused by Staphylococcus aureus, E. coli, Proteus spp., Klebsiella spp., Pseudomonas spp. and Coagulase negative staphylococci (Biadeglegne et al., 2009; Tesfahunegn et al., 2009; Gedebou et al., 1987). Similar results have been obtained in Ghana (Newman et al., 2006), Nigeria (Okesola \& Oni, 2000; Shittu et al., 2002) and Uganda (Anguzu \& Olila, 2007).

The widespread uses of antibiotics, together with the length of time over which they have been available have led to major problems of resistant organisms, contributing to morbidity and mortality. Antimicrobial resistance can increase complications and costs associated with procedures and treatment. Antimicrobial resistance among pathogens of wound infections is on the increase (Taiwo et al., 2002; Anguzu \& Olila, 2007; Okesola \& Kehinde, 2008). The control of wound infections has become more challenging due to widespread bacterial resistance to antibiotics and to a greater incidence of infections caused by methicillin-resistant $S$. aureus (MRSA) and polymicrobic flora (Akinjogunla et al., 2009).

The prevalence of different bacteria in infected wounds varies and the knowledge of prevalence in an institution cannot be extrapolated to others. Apart from inter-institutional variation, trans-institutional variation also exists (Sule et al., 2002). In Ethiopia few studies have been done on the prevalence and antimicrobial resistance patterns of wound infections (Gedebou et al., 1987; Mulu et al., 2006; Fantahun et al., 2009; Tesfahunegn et al., 2009; Yismaw et al., 2010). These studies indicated that many of the bacterial isolates showed high levels of resistance to amoxicillin, tetracycline, chloramphenicol, erythromycin while low levels of resistance to gentamicin, cloxacillin, norfloxacine and ciprofloxacin were documented (Taiwo et al., 2002; Yismaw et al., 2010). Studies conducted so far focused on inpatients however data on antimicrobial susceptibility from outpatients is scarce. Knowledge of the causative agents of wound infection in a specific geographic region will therefore be useful in the selection of antimicrobials for empiric therapy. The objective of the present study was to identify the etiologies of infections over the course of eight years and determine the antimicrobial susceptibilities of the pathogens.

\section{Material and methods}

\section{Study design}

A retrospective review of culture results of wound swabs performed from 2003 to 2010 at Dessie Regional Health Research Laboratory was done. Demographic variables, the organism isolated and the antimicrobial resistance patterns were collected from the registration records using a standard data collection form.

\section{Culture and identification}

The laboratory standard operation procedure showed that wound swabs were collected using sterile cotton swabs. Wound specimens were inoculated on blood agar (for gram-positive bacteria), mannitol salt agar (selective media for S. aureus) and MacConkey agar (for gram- 
negative bacteria) (Oxoid, England). The plates were incubated in atmosphere at $37^{\circ} \mathrm{C}$ for $24-48$ h. All positive cultures were identified by their characteristic appearance on their respective media, gram staining and confirmed by the pattern of biochemical reactions using the standard method (Cheesbrough, 2004). Members of the family enterobacteriaceae and other gramnegative rods were identified by indole production, $\mathrm{H}_{2} \mathrm{~S}$ production, citrate utilization, motility test, urease test, carbohydrate utilization tests and other tests. For gram-positive bacteria coagulase, DNase, catalase, bacitracin and other tests were used (Cheesbrough, 2004).

\section{Antimicrobial susceptibility tests}

According to the standard operational procedures, antimicrobial susceptibility tests were done on Mueller-Hinton agar (Oxoid, Hampshire, England) using Kirby Bauer disk diffusion method (Bauer et al., 1966). Morphologically identical 4-6 bacterial colonies from overnight culture were suspended in $5 \mathrm{ml}$ nutrient broth and incubated for 4 hours at $37^{\circ} \mathrm{C}$. Turbidity of the broth culture was equilibrated to match $0.5 \mathrm{McF}$ arland standards. The surface of Mueller Hinton agar plate was evenly inoculated with the culture using a sterile cotton swab. The antibiotic discs were applied the surface of the inoculated agar. After 18-24 hours of incubation, the diameter of growth inhibition around the discs were measured and interpreted as sensitive, intermediate or resistant according to Clinical and Laboratory Standards Institute (CLSI, 2006) (formerly known as National Committee for Clinical Laboratory Standards / NCCLS). The antimicrobial agents tested were: tetracycline $(30 \mu g)$, nitrofurantoin $(300 \mu g)$, erythromycin $(15 \mu g)$, chloramphenicol $(30 \mu \mathrm{g})$, gentamicin $(10 \mu \mathrm{g})$, ciprofloxacin $(5 \mu \mathrm{g})$, cephalotin $(30 \mu \mathrm{g})$, cotrimoxazole $(25 \mu \mathrm{g})$, ceftriaxone $(30 \mu \mathrm{g})$, norflaxocin $(10 \mu \mathrm{g})$, cloxacillin $(5 \mu \mathrm{g})$ and amoxicillin

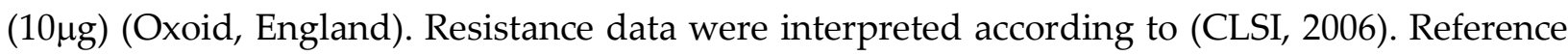
strains of E. coli ATCC 25922 and S. aureus ATCC 25923 were used for quality control for antimicrobial susceptibility tests.

\section{Statistical analysis}

Chi-square test was employed to compare the proportion of bacterial isolates with patient sex and age; and comparison of antimicrobial resistances. P-value of $<0.05$ was considered to indicate statistical significant difference.

\section{Ethical consideration}

Ethical approval was secured from Research Ethics Committee of Bahir Dar University. Permission from Dessie Regional Health Research Laboratory was also obtained.

\section{Results}

Between 2003 and 2010, a total of 599 wound swab samples analyzed. Four hundred twenty two $(70.5 \%)$ samples were culture positive while 177 (29.5\%) of wound swab cultures showed no growth (Table 1). A total of 500 bacteria were isolated from 422 cases (Table 2). Three hundred forty four $(81.5 \%)$ sample had single pathogens while $78(18.5 \%)$ had double infections. The 
number of isolates was higher than the number of cases because two pathogens were isolated from $78(18.5 \%)$ cases (Table 3).

Table 1: Age and sex distribution of patents with wound infections at Dessie regional laboratory, Ethiopia, from 2003 to 2010.

\begin{tabular}{llll}
\hline Demographic characteristics & & No. $(\%)$ of culture positive & P-value \\
\hline Age groups (years) & & & \\
& $<4(\mathrm{n}=16)$ & $11(68.8)$ & 0.01 \\
& $5-14(\mathrm{n}=71)$ & $49(69.0)$ & \\
$15-25(\mathrm{n}=205)$ & $151(73.7)$ & \\
& $26-44(\mathrm{n}=176)$ & $109(61.9)$ & \\
& $>44(\mathrm{n}=131)$ & $102(77.9)$ & 0.09 \\
& & \\
Gender & Male $(\mathrm{n}=368)$ & $271(64.2)$ & \\
& Female $(\mathrm{n}=231)$ & $151(35.8)$ & \\
& Total & $422(100)$ & \\
\end{tabular}

The age of the patients ranged from 1 year to 90 years, with mean age of $30.7(\mathrm{SD}=17.5)$ years. The mean age of male and female patients was [(31.2 (SD=18.1) and $29.8(\mathrm{SD}=16.3)]$ years, respectively. In this study males 368(61.4\%) constituted and females $231(38.6 \%)$ with male to female ratio of 1:1.6. Majority of the pathogens were isolated from males with isolation rate of $(64.2 \%)(\mathrm{CI}=0.45)$ and $35.8 \%(\mathrm{CI}=0.36)$, respectively (Table 1$)$.

Table 2: Type and frequency of pathogens isolated from wound infections at Dessie regional laboratory, Ethiopia, from 2003 to 2010.

\begin{tabular}{lll}
\hline Bacteria Isolated & Number $(\mathrm{n}=500)$ & Percent \\
\hline S. aureus & 208 & 41.6 \\
Pseudomonas spp. & 92 & 18.4 \\
E. coli & 82 & 16.4 \\
Proteus spp. & 55 & 11.0 \\
Enterobacter spp. & 21 & 4.2 \\
Citrobacter spp. & 21 & 4.2 \\
Klebsiella spp. & 12 & 2.4 \\
CNS & 9 & 1.8 \\
\hline Total & 500 & 100.0 \\
\hline
\end{tabular}

Key: CNS = Coagulase negative staphylococci

Table 2 illustrates the frequency of the pathogens isolated. Gram negative and gram positive bacteria constitute $56.6 \%$ and $43.4 \%$ of the bacterial isolates. S. aureus was the most frequently isolated pathogen which accounts for (41.6\%), followed by Pseudomonas spp., (18.4\%), E. coli (16.4\%), Proteus spp. (11.0\%), Enterobacter spp. and Citrobacter spp. (4.2\%), Klebsiella spp. $2.4 \%$ and CNS (1.8\%). 
S. aureus and Pseudomonas spp. were the most frequently isolated mixed infection (38.5\%). Infections with S. aureus and Proteus spp., S. aureus and E. coli, S. aureus and Citrobacter spp. with rates of $20.5 \%, 17.9 \% 9 \%$ and $6.4 \%$, respectively were among the double infections recorded in this study (Table 3 ).

Table 3: Type and number of pathogens in mixed wound infection at Dessie regional laboratory, Ethiopia, from 2003 to 2010

\begin{tabular}{lll}
\hline Pathogens & Frequency & Percentage \\
\hline S. aureus and Pseudomonas spp. & 30 & 38.5 \\
S. aureus and Proteus spp. & 16 & 20.5 \\
S. aureus and E. coli & 14 & 17.9 \\
S. aureus and Enterobacter spp. & 7 & 9.0 \\
S. aureus and Citrobacter spp. & 5 & 6.4 \\
Enterobacter spp. and Pseudomonas spp. & 3 & 3.8 \\
S. aureus and Klebsiella spp. & 1 & 1.3 \\
Klebsiella spp. and CNS & 1 & 1.3 \\
E.coli and Citrobacter spp. & 1 & 1.3 \\
\hline Total & 78 & 100 \\
\hline
\end{tabular}

Key: CNS = Coagulase negative staphylococci

Analysis of species specific resistance rates indicated that most of $S$. aureus was mostly resistant to amoxicillin $(79.1 \%)$ and tetracycline $(71.0 \%)$. On the other hand, S. aureus was susceptible to norflaxocin, ciprofloxacin, cloxacillin and gentamicin with resistance of only $8.0 \%, 8.8 \%, 10.3 \%$ and $12.4 \%$, respectively. Pseudomonas spp. showed the highest resistance to erythromycin $(90.5 \%)$, tetracycline $(90.3 .0 \%)$ and amoxicillin $(82.4 \%)$ while the resistance rates to ciprofloxacin and gentamicin were $4.6 \%$ and $7.8 \%$. E. coli isolates had resistant rates of $85.0 \%$ to amoxicillin and $71.4 \%$ to tetracycline whereas their resistance rates to ciprofloxacin and gentamicin were $7.7 \%$ and $14.4 \%$, respectively. Proteus spp. had resistance rate of $92.9 \%$ to erythromycin, $87.5 \%$ to tetracycline and $86.4 \%$ to amoxicillin. However, Proteus isolates were sensitive to gentamicin with resistance rate of only $9.6 \%$ and $16.7 \%$ resistance to ciprofloxacin (Table 4 ).

In this study, the overall multiple antimicrobial resistances rate was $65.2 \%$ and only $13 \%$ of the isolates were sensitive to eleven antimicrobial agents tested. The resistances against two or more antimicrobial agents were $47.6 \%, 82.6 \%, 75.6 \%$, and $87.3 \%$ for S. aureus, Pseudomonas spp., E. coli and Proteus spp., respectively (Table 5). 
Table 4: Susceptibility patterns of bacterial isolates from wound infections at Dessie regional laboratory, Ethiopia, 2003-2010

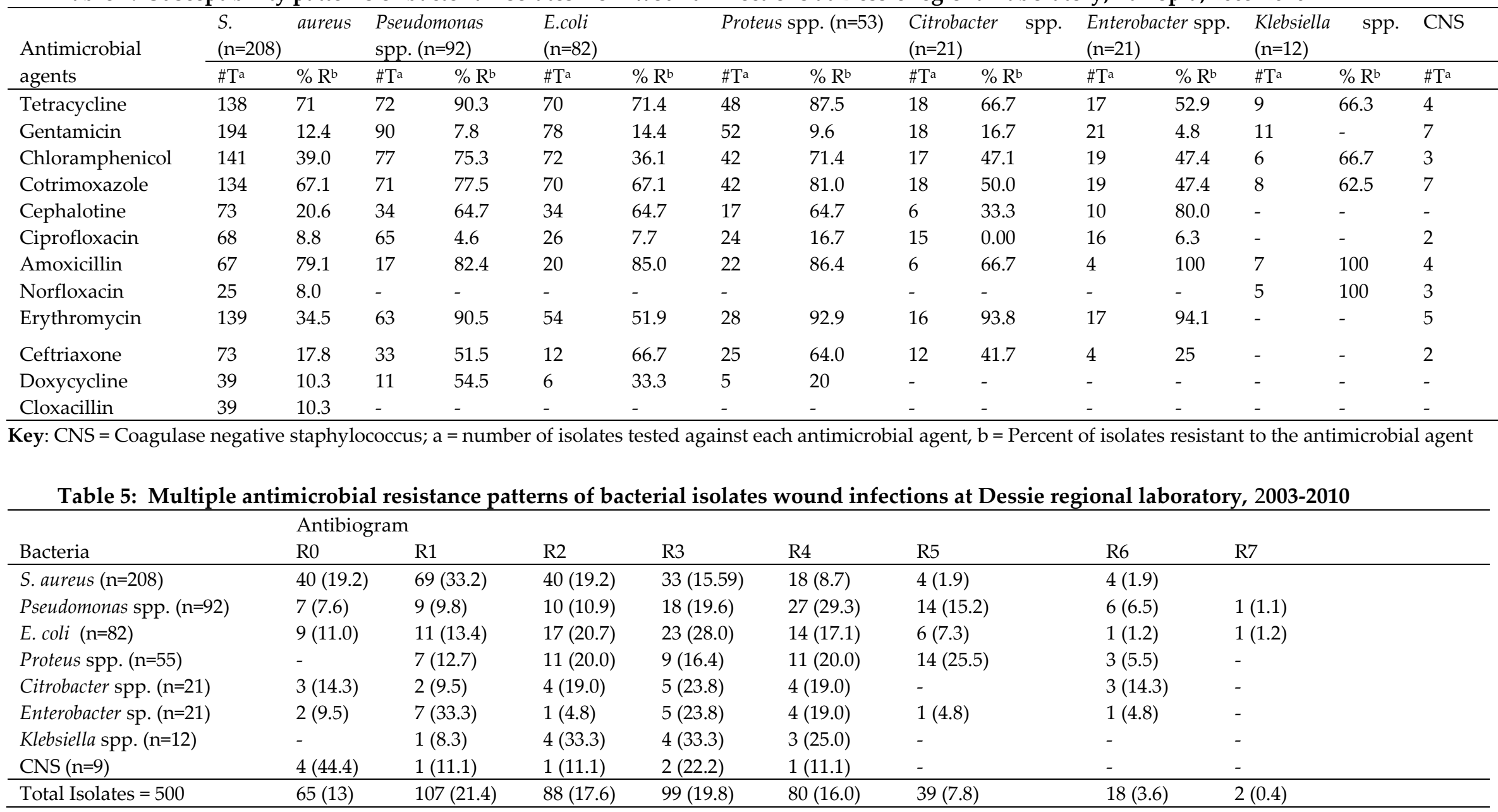

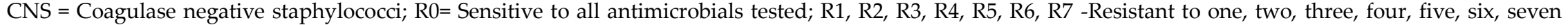
antimicrobials, respectively 


\section{Discussion}

Wound infections are responsible for significant mortality and morbidity, and incur substantial costs in hospitals. Antimicrobial resistance in wound pathogens is on the rise which can increase complications and costs associated with procedures and treatment (Bowler et al., 2001; Mulu et al., 2006; Wariso \& Nwachukwu, 2003). Identification of pathogens and determination of antibiograms from clinical specimens is beneficial to the patient and assists in selection of chemotherapy.

The isolation rate of pathogens in this study is higher compared to previous studied (Anguzu \& Olila, 2007; Mulu et al., 2006) however relatively lower than that reported from Ethiopia (Gizachew et al., 2010) and Nigeria (Wariso \& Nwachukwu, 2003). Statistically significant association was found between age of the patients and isolation rate of bacteria $(\mathrm{p}=0.01)$. Wariso \& Nwachukwu (2003) reported a similar result. In this study, as in several previous reports (Mulu et al., 2006; Gizachew et al., 2010; Wariso \& Nwachukwu, 2003; Surucuoglu et al., 2005) the most frequently isolated bacterium was $S$. aureus. It has been noted that S.aureus is the single causative bacterium in 25-655 of cutaneous abscess (Mahdi et al., 2000). Pseudomonas spp. was the second most common organism isolated followed by E. coli, Proteus spp., Enterobacter spp. and Citrobacter spp., Klebsiella spp. and CNS. The proportion of bacterial isolates was in agreement with several previous studies (Taiwo et al., 2002; Giacometti et al., 2000). Bacterial etiology can show geographic variations and may even vary over time within a population (Leegaard et al., 2000; Grude et al., 2001). Difference in identification methods are also known to influence the relative prevalence of bacteria which makes comparison of results difficult (Barret et al., 1999).

High resistance rates were recorded to amoxicillin, tetracycline and erythromycin. Significantly high resistance rate was demonstrated for amoxicillin, erythromycin and erythromycin $(\mathrm{p}<0.001)$. High level of resistance has been reported to tetracycline and erythromycin from studies conducted in Ethiopia and elsewhere (Mulu et al., 2006; Biadgelegne et al., 2009, Petkovšek et al., 2009). The sensitivity rates of norflaxocin, ciprofloxacin, cloxacillin and gentamicin were high. Gentamicin, ciprofloxacin, cloxacillin and norfloxacine were the most effective antimicrobials against most isolates. This finding is in line with the results documented from previous studies for ciprofloxacin (Anguzu \& Olila, 2007; Wariso \& Nwachukwu, 2003; Misra \& Yogesh, 2000), cloxacillin and gentamicin (Endalafer et al., 2011; Taiwo et al., 2002) and norfloxacin (Petkovšek et al., 2009). The relatively low level of resistance to these drugs can be because these drugs had been in the market for a relatively short period of time as compared to drugs such as tetracycline, amoxicillin and erythromycin (Neweman et al., 2006). Majority of the isolates $(65.2 \%)$ showed resistance to two or more antimicrobials. Previous studies in Ethiopia have demonstrated comparable results (Mulu et al., 2006; Yismaw et al., 2010).

This retrospective study is based on the results microbiological tests and susceptibility testing carried out between 2003 and 2010. Due to the nature of the retrospective analysis we couldn't trace patient's history. This study did not consider etiology of wound infections other 
than bacteria and anaerobic bacteria due to lack of facility. The number of antimicrobials were tested were also limited in some isolates.

In conclusion, the results of this study showed the prevalence and antimicrobial susceptibility patterns of wound infection over a period of eight years. The most frequently isolated bacteria were sensitive to ciprofloxacin, gentamicin, cloxacillin and norfloxacin. These antimicrobials are considered appropriate for empirical treatment of wound infections in the study area. Periodic monitoring of etiology and antimicrobial susceptibility both in the community and hospital settings is recommended.

\section{Acknowledgments}

We thank Mr. Fekadu Birru, head of Dessie Retinal Health Research Laboratory for supporting this research. We also thank all staff of microbiology department of Dessie Regional Health Research Laboratory for their proper documentation.

\section{References}

Akinjogunla, O. J., Adegoke, A. A., Mboto, C., Chukwudebelu, I. C. \& Udokang, I. (2009) Bacteriology of automobile accident wounds infection. International Journal of Medicine and Medical Sciences 1, 23-27.

Anguzu, J. R. \& Olila, D. (2007) Drug sensitivity patterns of bacterial isolates from septic post-operative wounds in a regional referral hospital in Uganda African Health Sciences 7, 148-154.

Barret, S.P., Savage, M.A., Rebec, M.P., Guyot, A., Andrde, N. \& Shrimpton, S.B. (1999) Antibiotic sensitivity of bacteria associated with community-acquired urinary tract infection in Britain. Journal of Antimicrobial Chemotherapy 44, 369-365.

Bauer, A.W., Kirby, W.M.M., Sherirs, J.C. \& Turck, M. (1966) Antibiotic susceptibility testing by standard single disk method. American Journal of Clinical Pathology 45, 433-496.

Biadgelegne, F., Abera, B., Alem, A. \& Anagaw, B. (2009) Bacterial isolates from wound infection and their

antimicrobial susceptibility pattern in Felege Hiwot Referral Hospital, Northwest Ethiopia. Ethiopian Journal of Health Sciences 19, 173-177.

Bowler, P. G. \& Davies, B. J. (1999) The microbiology of acute and chronic wounds. Wounds 11, 72-78.

Bowler, P.G., Duerden, B.I. \& Armstrong, D.G. (2001) Wound microbiology and associated approaches to wound management. Clinical Microbiology Reviews 14, 244-269.

Branom, R.N. (2002) Is this wound infected? Critical Care Nursing Quarterly 25, 55-62.

Cheesbrough, M. (2004) Medical laboratory manual for tropical countries. 2:146-180.

Clinical and Laboratory Standards Institute. (2007) Performance Standards for Antimicrobial Susceptibility Testing; Seventeenth Information Supplement. CLSI document M100-S17, Clinical and Laboratory Standards Institute Wayne Pennsylvania. 
Cutting, K.F. \& White, R. J. (2004) Defined and refined: criteria for identifying wound infection revisited. British Journal of Community Nursing 9, S6-S15.

Endalafer, N., Gebre-Selassie, S., \& Kotiso B. (2011) Nosocomial bacterial infections in a tertiary hospital in Ethiopia. Journal of Infection Prevention 12, 38-43

Giacometti, A., Cirioni, A. M., Schimizzi, M. S., Del Prete, F., Barchiesi, M. M., D’errico, E. P. \& Scalise, G. (2000) Epidemiology and microbiology of surgical wound infections. Journal of Clinical Microbiology 38, 918-922.

Gedebou, M., Kronvall, G., Habte-Gabr, E. \& Ringertz, S. (1987) The bacteriology of nosocomial infections at Tikur Anbessa Teaching Hospital, Addis Ababa. Acta Pathologica, Microbiologica et Immunologica Scandinavica (B) 95, 331-336.

Green, J.W. \& Wenzel, R.P. (1977) Postoperative wound infection: a controlled study of the increased duration of hospital stay and direct cost of hospitalization. Annals of Surgery 185, 264-268.

Grude, N., Tveten, Y. \& Kristianse, B.-E. (2001) Urinary tract infections in Norway: bacterial etiology and susceptibility. A retrospective study of clinical isolates. Clinical Microbiology and Infections 7, 543-547.

Larson, E. L., Gomez-Duarre, C., Lee, L. V., Kain, D. J. \& Keswick, B. H. (2002) Microbial flora of the hands of homemakers. American Journal Infection Control 31, 72-79.

Leegaard, T.M., Caugant, D.A., Froholm, L.O. \& Hoiby, E.A. (2000) Apparent difference in antimicrobial susceptibility as a consequence of national guidelines. Clinical Microbiology and Infection 6, 290.

Mahdi, S.I., Ahmed, A.A., Boelens, H, Ott, A., Abugroun, E.S., van-Belkum, A, Zijlstra, E., Verbrugh, H. \& Fahal. A. (2000) An epidemiological study of the occurrence of Staphylococcus aureus in superficial abscesses of patients presenting for surgery in a teaching hospital in Khartoum, Sudan. Immunology and Medical Microbiology 29, 155-162.

Misra, R.N. \& Yogesh, C. (2000) Antimicrobial resistance patterns of isolates from wound and soft tissue infections. Medical Journal of Armed Forces India 56, 205-208.

Mulu, A., Moges, F., Tessema, B. \& Kassu, A. (2006) Pattern and multiple drug resistance of bacterial pathogens isolated from wound infection at University of Gondar Teaching Hospital, Northwest Ethiopia. Ethiopian Medical Journal 44, 125-131.

Newman, M., J., Enoch, F., Asamoah-Adu, A. \& Sampane-Donkor, E. (2006) The GhanaianDutch Collaboration for Health Research and Development Project Number 2001/GD/07 2006; Technical Repot Series No. 5.

Okesola, A.O. \& Kehinde, A.O. (2008) Bacteriology of non-surgical wound infections in Ibadan, Nigeria. African Journal of Medicine and Medical Sciences 37, 261-264.

Okesola, A.O. \& Oni, A. A. (2009) Antimicrobial resistance among common bacterial pathogens in Southern Nigeria. American-Eurasia Journal of Agriculture and environmental Science 5, 327-330.

Petkovs`ek , Z., Elers`ič , K., Gubina, M., Z` gur-Bertok, D. \& Erjavec, M. S. (2009) Virulence Potential of Escherichia coli isolates from skin and soft tissue infections. Journal of Clinical Microbiology 47, 1811-1817. 
Shittu, A.O., Kolawole, D.O. \& Oyedepo, E.A.R. (2002) A study of wound infections in two health institutions in Ile-Ife, Nigeria. African Journal of Biomedical Research 5, 97-102.

Sule, A.M., Thanni, L.O.A., Sule-Odu, O.A., et al. (2002) Bacterial pathogens associated with infected wounds in Ogun State University Teaching Hospital, Sagamu, Nigeria. African Journal of Clinical and Experimental Microbiology. 3, 13-16.

Surucuoglu, S., Gazi, H., Kurutepe, S., Ozkutuk, N. \& Ozbakkaloglu, B. (2005) Bacteriology of surgical wound infections in a tertiary care hospital in Turkey. East African Medical Journal 82, 331-336.

Taiwo, S.S., Okesina, A.B. \& Onile, B. A. (2002) In Vitro Antimicrobial susceptibility patterns of bacterial isolates form wound infections in University of Ilorine Teaching Hospital. African Journal of Clinical and Experimental Microbiology 3, 6-10.

Tesfahunegn, Z., Asrat, D., Woldeamanuel, Y. \& Estifanos, K. (2009) Bacteriology of surgical site and catheter related urinary tract infections among patients admitted in Mekele Hospital,Mekele, Tigray, Ethiopia. Ethiopian Medical Journal 47, 117-112.

Wariso, B.A. \& Nwachukwu, C.O. (2003) A survey of common pathogens in wound in patients at the University of Port Harcourt Teaching Hospital (U.P.T.H), Port Harcourt. West African Journal of Medicine 221, 50-54.

Yismaw, G., Abay, S., Asrat, D., Yifru, S. \& Kassu, A. (2010) Bacteriological profile and resistant patterns of clinical isolates from pediatric patients, Gondar University Teaching Hospital, Gondar Northwest Ethiopia. Ethiopian Medical Journal 48(4), 293-300. 